\title{
RADIALLY SYMMETRIC SOLUTIONS OF A CLASS OF SINGULAR ELLIPTIC EQUATIONS
}

\author{
by JUAN A. GATICA* $\uparrow$, GASTON E. HERNANDEZ and P. WALTMAN $\dagger$
}

(Received 17th August 1988)

The boundary value problem

$$
\begin{gathered}
y^{\prime \prime}+\frac{N-1}{x} y^{\prime}+f(x, y)=0 \\
y^{\prime}(0)=0 \\
y(1)=0
\end{gathered}
$$

is studied with a view to obtaining the existence of positive solutions in $C^{1}([0,1]) \cap C^{2}((0,1))$. The function $f$ is assumed to be singular in the second variable, with the singularity modeled after the special case $f(x, y)=a(x) y^{-p}, p>0$.

This boundary value problem arises in the search of positive radially symmetric solutions to

$$
\begin{aligned}
\Delta u+f(|x|, u) & =0 \quad x \in \Omega \\
u / \Gamma & =0
\end{aligned}
$$

where $\Omega$ is the open unit ball in $\mathbb{R}^{N}$, centered at the origin, $\Gamma$ is its boundary and $|x|$ is the Euclidean norm of $x$.

1980 Mathematics subject classification (1985 Revision): $35 \mathrm{~J} 65$

\section{Introduction}

Radial symmetry occurs naturally in a variety of applied and geometric problems. Even when the problem is not inherently symmetric such solutions are often sought as the beginning of a perturbation argument (see also [9] for a different type of equation). Their place in the theory has taken new importance in view of the recent results of Gidas, $\mathrm{Ni}$ and Niremberg [6] and work in the direction of determining the existence of positive radially symmetric solutions to equations of the form

$$
\Delta u+f(|x|, u)=0,
$$

where $x \in \mathbb{R}^{N}$ and $|x|$ is the Euclidean norm, has appeared in $[1,3]$.

This paper deals with the existence and uniqueness of radially symmetric solutions of a nonlinear boundary value problem

$$
\begin{aligned}
\Delta u+f(|x|, u) & =0, \quad x \in \Omega \\
u / \Gamma & =0
\end{aligned}
$$

where $\Omega$ is open unit ball in $\mathbb{R}^{N}$ and $\Gamma$ is its boundary. The special case $f(|x|, u)=$

*This research was performed while the author was visiting Emory University.

tResearch supported by AFSOR 87-0140. 
$a(|x|) u^{-p}$, with $p$ in the open interval $(0,1)$, was considered in [5]. This type of nonlinearity for positive and negative exponents is well documented in physics $[1,2,7$, 8, 11, 12] (some of these papers base their applications on ordinary differential equations). The negative exponent gives a nonlinear singularity which seems to thwart the usual perturbation schemes or the more standard fixed point arguments; it should be noted, however, that singular problems of this type, for ordinary differential equations, have been studied in $[7,8,10,11]$, among others.

The search for radially symmetric solutions of (1.1) leads to the following problem in ordinary differential equations:

$$
\begin{gathered}
y^{\prime \prime}+\frac{N-1}{x} y^{\prime}+f(x, y)=0 \\
y^{\prime}(0)=0 \\
y(1)=0
\end{gathered}
$$

which is the problem to be studied in this paper.

Concerning the function $f(x, y)$, the following hypothesis will be adopted throughout:

(H-1) $f:[0,1) \times(0, \infty) \rightarrow(0, \infty)$ is continuous.

(H-2) $f(x, y)$ is decreasing in $y$ for each fixed $x$ and integrable over $[0,1]$ for each fixed $y$.

$$
\begin{gathered}
\lim _{y \rightarrow 0^{+}} f(x, y)=\infty \\
\lim _{y \rightarrow \infty} f(x, y)=0
\end{gathered}
$$

both limits being uniform on compact subsets of $(0,1)$.

$(\mathrm{H}-4)$ For every $\theta>0$

$$
\int_{0}^{1} f(x, \theta(1-x)) d x<\infty
$$

Note that in the case $f(x, y)=y^{-p}, 0<p<1$, the integral condition is trivially satisfied. In the case when $f(x, y)=a(x) y^{-p},(\mathrm{H}-4)$ holds for all $\theta>0$ if and only if it holds for $\theta=1$.

By a solution of (1.2) we mean a function $\phi$ in $C^{1}([0,1]) \cap C^{2}((0,1))$ which satisfies the boundary conditions and satisfies the differential equation on $(0,1)$. Furthermore, we are only interested in the existence of solutions to (1.2) which are positive on the interval $[0,1)$. 
The principal results take the form:

Theorem 1.1. Let ( $\mathrm{H}-1)-(\mathrm{H}-4)$ hold. Then Problem (1.2) has at least one positive solution. Furthermore, the set of positive solutions is bounded in the uniform norm.

Theorem 1.2. If, in addition to $(\mathrm{H}-1)-(\mathrm{H}-4), f(x, y)$ is locally Lipschitzian and strictly decreasing in $y$ then (1.2) has exactly one positive solution.

In the discussion that follows we will consider only the case $n=2$ but the general case follows in the same way. The basic idea is to convert Problem (1.2) into an integral equation of the form

$$
y(x)=\int_{0}^{1} G(x, t) f(t, y(t)) d t
$$

and seek a fixed point in an appropriate setting. Unfortunately, the fact that the function $f(x, y)$ is not defined for $y=0$ precludes a direct approach. Instead, one will replace $f(x, y)$ by $f_{n}(x, y)$ where $f_{n}$ has a certain "geometric" property. It will be possible to find a fixed point $\phi_{n}$ for (1.3) with $f$ replaced by $f_{n}$. The crucial arguments then involve estimates of the set of fixed points $\left\{\phi_{n}: n=1,2, \ldots\right\}$ from which a fixed point of (1.3) is obtained.

\section{The set of fixed points}

Let

$$
G(x, t)= \begin{cases}-t \ln (t), & 0<x \leqq t \\ -t \ln (x), & t \leqq x \leqq 1\end{cases}
$$

This is the Green function for the boundary value problem

$$
\begin{gathered}
y^{\prime \prime}+\frac{1}{x} y^{\prime}=0 \\
y^{\prime}(0)=0 \\
y(1)=0 .
\end{gathered}
$$

For $N>2$ one would use

$$
G(x, t)= \begin{cases}\frac{1}{N-2} t^{N-1}\left(t^{2-N}-1\right) & 0<x \leqq t \\ \frac{1}{N-2} t^{N-1}\left(x^{2-N}-1\right) & 0<t \leqq x \leqq 1\end{cases}
$$


Let $X$ be the Banach space of continuous functions defined on $[0,1]$ with the sup norm and $K$ the positive cone in $X$.

Define the set $D$ by

$$
D=\{\varphi \mid \varphi \in X, \exists \theta>0 \ni \varphi(x) \geqq \theta(1-x), x \in[0,1]\}
$$

and an operator $T: D \rightarrow K$ by

$$
T \varphi(x)=\int_{0}^{1} G(x, t) f(t, \varphi(t)) d t
$$

with $T \varphi(0)$ defined as an improper integral. This operator is continuous and for $\varphi \in D$

$$
(T \varphi)^{\prime}(x)=-\frac{1}{x} \int_{0}^{x} t f(t, \varphi(t)) d t, \quad x \in(0,1]
$$

and

$$
(T \varphi)^{\prime}(0)=0
$$

If $\varphi \in D, T \varphi$ is a strictly decreasing function and $(T \varphi)^{\prime}(1)<0$, so there exists a $\theta>0$ such that $(T \varphi)(x) \geqq \theta(1-x)$. Thus $T: D \rightarrow D$. (See [5] for a similar computation.) It follows also that $T \varphi$ is twice differentiable on $(0,1)$ with

$$
(T \varphi)^{\prime \prime}(x)=\frac{1}{x^{2}} \int_{0}^{x} t f(t, \varphi(t)) d t-f(x, \varphi(x))
$$

Lemma 2.1. If $\varphi \in D$ such that $T \varphi=\varphi$, then $\varphi \in C([0,1]) \cap C^{2}((0,1))$, and is a solution of (1.2). Furthermore, if $\varphi$ is any positive solution of $(1.2)$ in $C^{1}([0,1]) \cap C^{2}((0,1))$, then $\varphi \in D$ and $T \varphi=\varphi$.

Thus Theorem 1.1 will be proved if a fixed point of $T$ can be found in $D$. If $\varphi_{1} \varphi_{2} \in D$ then the closed order interval, denoted $\left\langle\varphi_{1}, \varphi_{2}\right\rangle$, is also contained in $D$ and $T$ is compact on $\left\langle\varphi_{1}, \varphi_{2}\right)$ (Ascoli).

Define a sequence of functions $\psi_{n}$ on $[0,1]$ by

$$
\psi_{n}(x)=\int_{0}^{1} G(x, t) f(t, n) d t, \quad n=1,2, \ldots
$$

Note that $\psi_{n}(x)>0$ for $x \in[0,1), \psi_{n+1}(x) \leqq \psi_{n}(x)$ and $\lim _{n \rightarrow \infty} \psi_{n}(x)=0$, uniformly on compact subsets of $(0,1)$. Given the sequence $\left\{\psi_{n}(x)\right\}$ define a sequence of functions of two variables by

$$
f_{n}(x, y)=f\left(x, \max \left(y, \psi_{n}(x)\right)\right.
$$


and a sequence of mappings $T_{n}: K \rightarrow K$ by

$$
\left(T_{n} \varphi\right)(x)=\int_{0}^{1} G(x, t) f_{n}(t, \varphi(t)) d t
$$

Note that $f_{n}:(0,1) \times[0, \infty) \rightarrow(0, \infty)$ and $T_{n} K \subset D, n=1,2, \ldots T_{n}$ is compact (since $\left(T_{n} \varphi\right)^{\prime}$ is bounded on any bounded subset of $K), T_{n}(0) \geqq 0$, and $T_{n}^{2}(0) \geqq 0$.

Lemma 2.2 [5]. Let $X$ be a Banach space, $K$ a normal cone in $X, D$ a subset of $K$ such that if $x, y$ are elements of $D, x \leqq y$, then $\langle x, y\rangle$ is contained in $D$. Let $T: D \rightarrow K$ be a decreasing mapping which is compact on any closed order interval contained in $D$. If there exists $x_{0} \in D$ such that $T^{2} x_{0}$ is defined and $T x_{0}, T^{2} x_{0}$ are such that $T x_{0} \geqq x_{0}, T^{2} x_{0} \geqq x_{0}$, then $T$ has a fixed point.

Since the nonnegative cone in $X$ is normal, Lemma 2.2 implies that $T_{n}$ has a fixed point, $\varphi_{n}$, which belongs to $K$. The hypotheses on $f$ yield that $\varphi_{n}(x)>0$ for $x \in[0,1)$. It is from this sequence of fixed points of the operators $T_{n}$ that a solution will be obtained. The important properties of the set of fixed points $\left\{\varphi_{n}\right\}$ are contained in the next section.

\section{Two properties of the set of fixed points}

In this section we provide two technical lemrnas which are important in carrying out the proof of Theorem 1.1.

Lemma 3.1. The set of fixed points $\left\{\varphi_{n}\right\}$ satisfies

$$
\varphi_{n}(0) \leqq \varphi_{n+1}(0), \quad n=1,2, \ldots
$$

Lemma 3.2. There exists an $R>0$ such that $\left\|\varphi_{n}\right\| \leqq R, n=1,2, \ldots$.

Proof of Lemma 3.1. Suppose to the contrary $\varphi_{n+1}(0)<\varphi_{n}(0)$ for some $n$, and hence $\varphi_{n+1}(x)<\varphi_{n}(x)$ immediately to the right of zero, say on $[0, \delta)$. Since

$$
\begin{aligned}
\varphi_{n}^{\prime \prime}(x) & =-f_{n}\left(x, \varphi_{n}(x)\right) \geqq-f_{n+1}\left(x, \varphi_{n}(x)\right) \\
& \geqq-f_{n+1}\left(x, \varphi_{n+1}(x)\right)=\varphi_{n+1}^{\prime \prime}(x), \quad x \in[0, \delta),
\end{aligned}
$$

and $\varphi_{n}^{\prime}(0)=\varphi_{n+1}^{\prime}(0)$, it follows that $\varphi_{n+1}(x)<\varphi_{n}(x)$ on $[0,1]$. However, since

$$
\begin{aligned}
\varphi_{n}(x) & =\int_{0}^{1} G(x, t) f_{n}\left(x, \varphi_{n}(x)\right) d x \\
& \leqq \int_{0}^{1} G(x, t) f_{n+1}\left(x, \varphi_{n+1}(x)\right) d t=\varphi_{n+1}(x),
\end{aligned}
$$


this is a contradiction.

Proof of Lemma 3.2. Suppose to the contrary that

$$
\lim _{n \rightarrow \infty}\left\|\varphi_{n}\right\|=\lim _{n \rightarrow \infty} \varphi_{n}(0)=\infty
$$

Let $u_{n}(x)=x \varphi_{n}(x)$ and note that $u_{n}$ is a function which is concave down with $u_{n}(0)=u_{n}(1)=0, u_{n}^{\prime}(0)=\varphi_{n}(0)$. As in [10] (see also [4]), $u_{n}(x) \geqq \alpha u_{n}\left(z_{n}\right)$ for $x \in[\alpha, 1-\alpha]$ for any $\alpha$ between 0 and $1 / 2$, where $z_{n}$ is the unique maximum of $u_{n}(x)$. For $x \in[\alpha, 1-\alpha]$,

$$
\frac{\alpha}{1-\alpha}\left\|u_{n}\right\| \leqq \varphi_{n}(x) \leqq \frac{\left\|u_{n}\right\|}{\alpha}
$$

so that $\lim _{n \rightarrow \infty}\left\|u_{n}\right\|=\infty$. By $(H-3)$ one can choose $N_{1}=N_{1}(\alpha)$ such that $f(x, y) \leqq 1$ for $x \in[\alpha, 1-\alpha]$ and $y \geqq N_{1}$. Since $\left\|u_{n}\right\| \rightarrow \infty$ for each fixed $\alpha$ there exists $N_{2}(\alpha)$ such that $(\alpha / 1-\alpha)\left\|u_{n}\right\| \geqq N_{1}$ for $n \geqq N_{2}$, and hence $\varphi_{n}(x) \geqq N_{1}$ for $x \in[\alpha, 1-\alpha]$ for $n \geqq N_{2}$.

From the definition of $\varphi_{n}$ one has that

$$
\begin{aligned}
\varphi_{n}(x) & =T_{n} \varphi_{n}(x) \\
& \leqq T \varphi_{n}(x) \\
& =\int_{0}^{\alpha} G(x, t) f\left(t, \varphi_{n}(t) d t+\int_{\alpha}^{1-\alpha} G(x, t) f\left(t, \varphi_{n}(t)\right) d t+\int_{1-\alpha}^{1} G(x, t) f\left(t, \varphi_{n}(t)\right) d t .\right.
\end{aligned}
$$

The middle integral is easily estimated for $n$ sufficiently large (by the above argument) by $M(\alpha)[1-2 \alpha]$ where $M(\alpha)$ is the maximum of $G$ on $[\alpha, 1-\alpha] \times[\alpha, 1-\alpha]$.

On $[1-\alpha, 1]$ it follows that $\left.u_{n}(x) \geqq(1-x) / \alpha\right) u(1-\alpha)$ since $u$ is concave down. Hence:

$$
\begin{aligned}
\varphi_{n}(x) & \geqq \frac{1-\alpha}{\alpha} \varphi_{n}(1-\alpha)\left(\frac{1-x}{x}\right) \\
& \geqq \frac{1-\alpha}{\alpha}\left(\frac{\alpha}{1-\alpha}\left\|u_{n}\right\|\right)\left(\frac{1-x}{x}\right) \\
& \geqq \frac{1-\alpha}{\alpha} N_{1}(1-x) \\
& =K(1-x)
\end{aligned}
$$

for $n \geqq N_{2}$. 
Thus

$$
\begin{aligned}
\int_{1-\alpha}^{1} G(x, t) f\left(t, \varphi_{n}(t)\right) d t & \leqq \int_{1-\alpha}^{1} G(x, t) f(t, K(1-t)) d t \\
& \leqq \int_{0}^{1} G(x, t) f(t, K(1-t)) d t \\
& <\infty
\end{aligned}
$$

by hypothesis.

To estimate the integral on $[0, \alpha]$ let us recall that by Lemma 3.1 we have $\varphi_{n}(0) \leqq \varphi_{n+1}(0)$ and we are assuming that $\lim _{n \rightarrow \infty} \varphi_{n}(0)=\infty$. We will assume, without loss of generality, that there exists $n_{0}>0$ such that $\varphi_{n}(0)>n_{0}+1$ and for each $n \in \mathbb{N}$ we will let $\eta_{n}$ be the unique element of $(0,1)$ for which $\varphi_{n}\left(\eta_{n}\right)=1$. If it should happen that $\inf _{n \in \mathbb{N}} \eta_{n}>0$ then we pick $\alpha=\inf _{n \in N} \eta_{n}$ and observe that by the continuity of $f, f(t, 1)$ must be bounded (as a function of $t$ ) in $[0, \alpha]$ and therefore, for $n \in \mathbb{N}$

$$
\int_{0}^{\alpha} G(x, t) f\left(t, \varphi_{n}(t)\right) d t \leqq \int_{0}^{\alpha} G(x, t) f(t, 1) d t
$$

obtaining the contradiction that the functions $\varphi_{n}$ must be uniformly bounded.

The only case left is when $\inf _{n \in N} \eta_{n}=0$. In this case, again by going to a subsequence if necessary, it may be assumed without loss of generality that $\eta_{n} \rightarrow 0$ monotonically. Then the following must occur:

$$
\begin{aligned}
\varphi_{n}^{\prime \prime}+\frac{1}{x} \varphi_{n}^{\prime}(x) & =-f_{n}\left(x, \varphi_{n}(x)\right) \\
& \geqq-f\left(x, \varphi_{n}(x)\right) \\
& \geqq-f(x, 1), \quad \text { for } 0 \leqq x \leqq \eta_{n} .
\end{aligned}
$$

Thus:

$$
\left(x \varphi_{n}^{\prime}(x)\right)^{\prime} \geqq-x f(x, 1)
$$


and

$$
x \varphi_{n}^{\prime}(x) \geqq-\int_{0}^{x} t f(t, 1) d t, \quad \text { for } 0 \leqq x \leqq \eta_{n}
$$

Thus:

$$
\begin{aligned}
\varphi_{n}^{\prime}(x) & \geqq-\int_{0}^{x} \frac{t}{x} f(t, 1) d t \\
& \geqq-\int_{0}^{x} f(t, 1) d t \\
& \geqq-\int_{0}^{1} f(t, 1) d t=-c, \quad \text { for } 0 \leqq x \leqq \eta_{n} .
\end{aligned}
$$

Observe that $c$ is independent of $n$. Now one has:

$$
\varphi_{n}(0)-\varphi_{n}\left(\eta_{n}\right)=-\varphi_{n}^{\prime}\left(\gamma_{n}\right) \eta_{n}
$$

for some $\gamma_{n}$ between 0 and $\eta_{n}$. Since $\varphi_{n}(0)>n_{0}+1$, we get

$$
n_{0}<-\varphi_{n}^{\prime}\left(\gamma_{n}\right) \eta_{n}
$$

or

$$
\varphi_{n}^{\prime}\left(\gamma_{n}\right)<\frac{-n_{0}}{\eta_{n}}
$$

Choosing $n$ so large as to have $\left(n_{0} / \eta_{n}\right)>c$ yields a contradiction.

Since this was the last remaining case, the proof of Lemma 3.2 is complete.

\section{Proof of the main results}

Proof of Theorem 1.1. Note first that

$$
\begin{aligned}
\varphi_{n}(x) & =T_{n} \varphi_{n}(x) \\
& =\int_{0}^{1} G(x, t) f_{n}\left(t, \varphi_{n}(t)\right) d t \\
& \geqq \int_{0}^{1} G(x, t) f_{n}(t, R) d t
\end{aligned}
$$




$$
\geqq \int_{0}^{1} G(x, t) f_{1}(t, R) d t
$$

where $R>0$ is given by Lemma 3.2 .

Define $\mathscr{X}:[0,1] \rightarrow[0, \infty]$ by

$$
\mathscr{X}(x)=\int_{0}^{1} G(x, t) f_{1}(t, R) d t
$$

and note that $\mathscr{X} \in D$ and hence there exists a $\theta_{0}>0$ such that $\mathscr{X}(x) \geqq \theta_{0}(1-x)$. Abusing notation, we let $R$ denote the constant function on $[0,1]$ so that $\left\langle g_{\theta_{0}}, R\right\rangle$ is an order interval where $g_{\theta_{0}}(x)=\theta_{0}(1-x)$. Clearly $\left\langle g_{\theta_{0}}, R\right\rangle$ is contained in $D$ and $g_{\theta_{0}} \leqq \varphi_{n} \leqq R$, $n=1,2, \ldots$. Since $T$ is compact on order intervals contained in $D,\left\{T \varphi_{n}\right\}_{n=1}^{\infty}$ contains a convergent subsequence (which we again label $\left\{T \varphi_{n}\right\}_{n=1}^{\infty}$ ) so

$$
\lim _{n \rightarrow \infty} T \varphi_{n}=\varphi^{*}
$$

with $\varphi^{*} \in D$.

This $\varphi^{*}$ will be a solution to (1.2). One must show that

$$
T \varphi^{*}=\varphi^{*}
$$

Since $\lim _{n \rightarrow \infty} T \varphi_{n}=\varphi^{*}$, one needs only that $\varphi_{n}(x)$ converges to $\varphi^{*}(x)$. This is contained in the next lemma whose proof we defer until the end of the proof of Theorem 1.1.

Lemma 4.1. $\varphi_{n}(x)$ converges to $\varphi^{*}(x)$ (uniformly on compact subsets of $(0,1)$ ).

Proof of Theorem 1.1 (continued). Applying $T$ to $\varphi^{*}$ yields that

$$
\begin{aligned}
T \varphi^{*}(x) & =\int_{0}^{1} G(x, t) f\left(t, \varphi^{*}(t)\right) d t \\
& =\int_{0}^{1} G(x, t) f\left(t, \lim \varphi_{n}(t)\right) d t \\
& =\int_{0}^{1} G(x, t) \lim _{n \rightarrow \infty} f\left(t, \varphi_{n}(t)\right) d t .
\end{aligned}
$$

Since $\varphi_{n}(x) \geqq \theta_{0}(1-x), f(t, y)$ is decreasing in $y$, and for each fixed $y$,

$$
\int_{0}^{1} G(x, t) f\left(t, \theta_{0}(1-t)\right) d t<\infty,
$$


the dominated convergence theorem allows one to take the limit outside the integral sign. Hence,

$$
\begin{aligned}
T \varphi^{*}(x) & =\lim _{n \rightarrow \infty} \int_{0}^{1} G(x, t) f\left(t, \varphi_{n}(t)\right) d t \\
& =\lim _{n \rightarrow \infty} T \varphi_{n}(x) \\
& =\varphi^{*}(x) .
\end{aligned}
$$

Thus, $\varphi^{*}$ is the desired fixed point of $T$ and the proof of existence in Theorem 1.1 is complete (as soon as a proof of Lemma 4.1 is given).

The proof of the boundedness (in the supremum norm) of the set of positive solutions proceeds in the same way as the proof of Lemma 3.2.

Proof of Lemma 4.1. Let $C \subseteq(0,1)$ be compact and let $\varepsilon>0$. Pick $\alpha \in(0,1 / 2)$ such that $C \subseteq[\alpha, 1-\alpha]$ and such that

$$
-2\left[\ln (\alpha) \int_{0}^{\alpha} t f\left(t, \theta_{0}(1-t)\right) d t+\int_{1-\alpha}^{1} t \ln (t) f\left(t, \theta_{0}(1-t)\right) d t\right]<\varepsilon
$$

Pick $M$ such that for $n \geqq M \psi_{n}(x) \leqq \theta_{0}(1-x), x \in[\alpha, 1-\alpha]$. For $n \geqq M$ and $x \in[\alpha, 1-\alpha]$ one sees that

$$
\begin{aligned}
T \varphi_{n}-\varphi_{n}(x)= & \int_{0}^{1} G(x, t)\left[f\left(t, \varphi_{n}(t)\right)-f\left(t, \max \left(\varphi_{n}(t), \psi_{n}(t)\right)\right)\right] d t \\
= & \int_{0}^{\alpha} G(x, t)\left[f\left(t, \varphi_{n}(t)\right)-f\left(t, \max \left(\varphi_{n}(t), \psi_{n}(t)\right)\right] d t\right. \\
& +\int_{1-\alpha}^{1} G(x, t)\left[f\left(t, \varphi_{n}(t)\right)-f\left(t, \max \left(\varphi_{n}(t), \psi_{n}(t)\right)\right] d t .\right.
\end{aligned}
$$

Thus:

$$
\begin{aligned}
\left|T \varphi_{n}(x)-\varphi_{n}(x)\right| & \leqq 2\left[\int_{0}^{\alpha} G(x, t) f\left(t, \varphi_{n}(t)\right) d t+\int_{-\alpha}^{1} G(x, t) f\left(t, \varphi_{n}(t)\right) d t\right] \\
& \leqq 2\left[\int_{0}^{\alpha} G(x, t) f\left(t, \theta_{0}(1-t)\right) d t+\int_{1-\alpha}^{1} G(x, t) f\left(t, \theta_{0}(1-t)\right) d t\right] .
\end{aligned}
$$


Since $x \in[\alpha, 1-\alpha]$, for $t \in(0, \alpha]$ we must have

$$
G(x, t)=-t \ln (x) \leqq-t \ln (\alpha)
$$

and for $t \in[1-\alpha, 1]$,

$$
G(x, t)=-t \ln (t)
$$

Thus:

$$
\begin{aligned}
\left|T \varphi_{n}(x)-\varphi_{n}(x)\right| & \leqq-2\left[\ln (\alpha) \int_{0}^{\alpha} t f\left(t, \theta_{0}(1-t)\right) d t+\int_{1-\alpha}^{1} t \ln (t) f\left(t, \theta_{0}(1-t)\right) d t\right] \\
& <\varepsilon
\end{aligned}
$$

Since $C \subseteq(0,1)$ was an arbitrary compact set, the proof of Lemma 4.1 is complete.

Proof of Theorem 1.2. Suppose that there are two distinct solutions of (1.2), labelled $\varphi_{1}(x)$ and $\varphi_{2}(x)$. Observe that the Lipschitz condition implies that the initial value problem is well posed for any $x_{0} \in(0,1)$ and

$$
\begin{array}{r}
y\left(x_{0}\right)=\alpha>0 \\
y^{\prime}\left(x_{0}\right)=\beta \leqq 0 .
\end{array}
$$

This implies that $\varphi_{1}$ is not equal to $\varphi_{2}$ on any interval. Furthermore, $\varphi_{1}(x)>\varphi_{2}(x)$ for all $x \in(0,1)$ is not possible, since

$$
\begin{aligned}
\varphi_{1}(x) & =\int_{0}^{1} G(x, t) f\left(t, \varphi_{1}(t)\right) d t \\
& <\int_{0}^{1} G(x, t) f\left(t, \varphi_{2}(t)\right) d t=\varphi_{2}(x) .
\end{aligned}
$$

Hence $\varphi_{1}(x)$ and $\varphi_{2}(x)$ must cross in $(0,1)$. Let $0<x_{1} \leqq x_{2} \leqq 1$ be any two consecutive "crossings" with $\varphi_{2}(x)>\varphi_{1}(x)$ for $x_{1}<x<x_{2}$. Then if $w(x)=\varphi_{2}(x)-\varphi_{1}(x)$ we have that $w(x)>0$ on $\left(x_{1}, x_{2}\right)$ and $w\left(x_{1}\right)=w\left(x_{2}\right)=0$, concluding that there must exist a point of maximum $\xi \in\left(x_{1}, x_{2}\right)$. At any such point of maximum:

$$
\begin{aligned}
w^{\prime \prime}(\xi) & =\varphi_{2}^{\prime \prime}(\xi)-\varphi_{1}^{\prime \prime}(\xi) \\
& =f\left(\xi, \varphi_{1}(\xi)\right)-f\left(\xi, \varphi_{2}(\xi)\right) \\
& >0
\end{aligned}
$$


and this contradicts the fact that $\xi$ is a maximum.

This completes the proof of Theorem 1.2.

\section{REFERENCES}

1. F. V. Atkinson and L. A. Peletier, Ground states of $-\Delta u=f(u)$ and the Emden-Fowler equation, Arch. Rational Mech. Anal. 93 (1986), 103-107.

2. J. D. Diaz, J. M. Morel and L. Oswald, An elliptic equation with singular nonlinearity, Comm. Partial Differential Equations 12 (1987), 1333-1344.

3. L. Erbe and K. Sсhmitt, On radial solutions of some semilinear elliptic equations, Diff. and Int. Equations 1 (1988), 71-78.

4. J. A. Gatica, V. Oliker and P. Waltman, Iterative procedures for nonlinear second order boundary value problems (Emory University preprint, 1987).

5. J. A. Gatica, V. Oliker and P. Waltman, Singular nonlinear boundary value problems for second order differential equations, J. Differential Equations 79 (1989), 62-78.

6. B. Gidas, Wei-Ming Ni and L. Niremberg, Symmetry of positive solutions of nonlinear elliptic equations in $\mathbb{R}^{n}$, Adv. Math Suppl. Stud. 7A (1981), 369-402.

7. A Nachman and A. Callegari, A nonlinear boundary value problem in the theory of pseudoplastic fluids, SIAM J. Appl. Math. 38 (1980), 275-281.

8. R. D. Nussbaum and C. A. Stuart, A singular bifurcation problem, J. London Math. Soc. (2) 14 (1976), 31-38.

9. V. I. OLIKER, Near radially symmetric solutions of an inverse problem in geometric optics, Inverse Problems 3 (1987), 743-756.

10. C. A. Stuart, Concave solutions of singular non-linear differential equations, Math. Z. 136 (1974), 117-135.

11. S. Taliaferro, A nonlinear singular boundary value problem, Nonlinear Anal. 3 (1979), 897-904.

12. J. S. W. Wong, On the generalized Emden-Fowler equation, SI AM Rev. 17 (1975), 339-360.

J. A. Gatica and G. E. Hernandez

Department of Mathematics

UNIVERSITY OF IOWA

Iowa City, IOWA 52242

U.S.A.
P. WALTMAN

Department of Mathematics and Computer Science

EMORY UNIVERSITY

Atlanta, Georgia 30322 